\title{
Detección de las mutaciones katG e inhA para guiar el uso de isoniazida y etionamida en el tratamiento de la tuberculosis multidrogorresistente en el Perú
} multidrug-resistant tuberculosis in Peru

Correspondencia Samuel Pecho-Silva samuelpechosilva@gmail.com

Recibido: $14 / 07 / 2020$ Aprobado: 20/08/2020

Citar como: Pecho-Silva S, Navarro-Solsol AC. Detección de las mutaciones katG e inhA para guiar el uso de isoniazida y etionamida en el tratamiento de la tuberculosis multidrogorresistente en el Perú. Acta Med Peru. 2020;37(4):559-61. doi: 10.35663/amp.2020.374.1049

\author{
Samuel Pecho-Silva ${ }^{1,2, a}$, Ana C. Navarro-Solsol ${ }^{3, b}$ \\ Universidad Científica del Sur. Lima, Perú. \\ Hospital Nacional Edgardo Rebagliati Martins. Lima, Perú. \\ Universidad Nacional de Ucayali. Pucallpa, Perú. \\ Médico cirujano especialista en Neumología; ${ }^{\mathrm{b}}$ médico cirujano
}

Sr. Editor,

La isoniazida (INH), desde su descubrimiento en la década de 1950, se constituyó en uno de los medicamentos fundamentales en el tratamiento de la tuberculosis (TB). La actividad bactericida de la INH está dada por la inhibición de la biosíntesis del ácido micólico. La INH es activada por la catalasa peroxidasa sintetizada por el gen KatG y logra su efecto uniéndose al gen InhA del Mycobacterium tuberculosis. A partir de su primer uso, como monoterapia en 1952, se informó rápidamente del desarrollo de resistencia a INH (R-INH). Esta ocurre principalmente por una mutación en dos genes (el katG y/o el inhA). La mutación en el katG confiere R-INH de "alto nivel"; es decir, que la INH se hace ineficaz incluso a altas dosis (HAD) de $15-20 \mathrm{mg} / \mathrm{kg} /$ día, aunque se asocia a una sensibilidad de la bacteria a la etionamida (ETO). La mutación del gen inhA, por su parte, causa una resistencia de "bajo nivel", lo que significa que la HAD podría ser efectiva como parte del tratamiento, pero le confiere resistencia a la ETO. El estudio de la mutación del gen que confiere R-INH, así como la frecuencia de las mutaciones ayuda a tomar decisiones clínicas al momento de diseñar un esquema de tratamiento ${ }^{[1-4]}$.

La frecuencia de estas dos mutaciones (KatG e InhA) varía geográficamente. La mutación katG suele ser más frecuente (entre el 42 al 95\% de los aislamientos) mientras que la de inhA ocurre entre un $5,4 \%$ a $8,4 \%$ de los aislamientos; además, entre un $10 \%$ y $16,8 \%$ de los aislamientos puede tener ambas mutaciones. En el Perú, la prueba que puede detectar la R-INH y la mutación responsable es el Genotype ${ }^{\circledR}$ MTBDRplus (Hain Lifescience, Nehren, Germany), que es realizada en el Instituto Nacional de Salud del Ministerio de Salud del Perú (MINSA). El método GeneXpert MTB/RIF (Cepheid, Estados Unidos) no detecta la R-INH ${ }^{[5-8]}$.

La prevalencia de R-INH global en 156 países ( $n=211753$ pacientes) se estima en 7,4\% (intervalo de confianza al 95\% [IC 95\%]: 6,5\% - 8,4\%) para los pacientes nuevos y en $11,4 \%$ (IC 95\%: 9,4\% - 13,4\%) para los pacientes antes tratados. La R-INH en el Perú para los pacientes nuevos está entre el 15,2\% y el $18,6 \%$ y en los tratados previamente entre el $19,2 \%$ y el $29,3 \%$, con un promedio del $17,9 \%$ en todos los pacientes con TB, constituyéndose en un serio problema de salud pública a nivel nacional ${ }^{[6,8]}$. 
Nosotros realizamos una revisión de los resultados de las pruebas de sensibilidad (PS) de los pacientes atendidos en el Programa de Control de la TB (PCT) del Hospital Nacional Edgardo Rebagliati Martins (HNERM) de la Seguridad Social del Perú (EsSalud), realizadas durante el periodo de setiembre 2017 a marzo del 2018. EI HNERM recibe a pacientes con TB que son referidos para su atención debido a que, entre otros motivos, tienen una PS con resultado anormal.

Las PS correspondieron a 331 pacientes atendidos en este periodo, con un promedio de edad de $45,5 \pm 18,6$ años, de los cuales el $61 \%$ fueron varones. El $78,54 \%(n=260)$ de los pacientes contaban con el resultado de al menos una PS. Se identificaron 24 pruebas MODS, 226 pruebas Genotype ${ }^{\circledR}$ MTBDRplus y 173 pruebas convencionales (proporciones y BACTEC/MGIT 960). Nueve PS MODS, 117 Genotype ${ }^{\circledR}$ y 117 convencionales expresaron TB multidrogorresistente (TBMDR), respectivamente. Doce expresaron TB extremadamente resistente (TB XDR, todos ellos en VIH-no reactivo y 9 de ellos eran pacientes nuevos) y 36 expresaron TB pre-XDR (21 por resistencia a kanamicina, cinco por resistencia a levofloxacino y 10 por resistencia a capreomicina).

De las 226 PS por Genotype ${ }^{\circledR}, 180$ correspondieron a R-INH, el $64,4 \%$ ( $n=116)$ por mutación del KatG; sólo dos $(1,11 \%)$ por una mutación simultánea KatG/InhA y las restantes $(34,44 \%)$ por mutación del InhA. En el caso de TB MDR por Genotype ${ }^{\circledR}$, el $71 \%(n=83)$ fue por mutación del KatG y de estos, el $80,72 \%$ fue sensible simultáneamente a ETO. Sólo 18,8\% $(n=22)$ de los casos TB MDR tuvieron mutación InhA y de ellos sólo el $60 \%$ fue sensible a HAD y ETO simultáneamente (Tabla 1).

El registro y estratificación del resultado de la PS es parte habitual del manejo médico de estos pacientes y las PS son procesadas en diferentes laboratorios. El número de muestras evaluadas $(n=226)$, correspondientes a un periodo de seis meses, representan el $72 \%$ del total de muestras que se pudieron obtener en cuatro años en Brasil y es un $45 \%$ mayor al total de muestras que obtuvieron en Mozambique, en un periodo de dos años ${ }^{[2]}$. Los resultados que hemos obtenido se correlacionan con lo encontrado en Mozambique en donde el $84,2 \%$ de muestras fueron R-INH por el gen KatG y un 10,5\% por el gen InhA. En Brasil se encontró un 54,5\% de R-INH por KatG y un 40,9\% por InhA ${ }^{[2]}$. A diferencia de lo reportado por Bollela et al. ${ }^{[2]}$, nosotros hemos podido correlacionar la R-INH con la HAD y la resistencia a ETO.

Estos resultados tienen las siguientes implicancias clínicas: 1) sólo un 18,8\% de pacientes con TB MDR se beneficiarían de HAD como parte del tratamiento para la TB MDR; 2) sólo un $67,28 \%$ de pacientes con TB MDR se beneficiarían de ETO como parte del tratamiento para la TB MDR; 3) sólo un $12,64 \%$ de pacientes con TB MDR se beneficiarían del esquema de tratamiento para TB MDR "acortado", que incluye a HAD y ETO propuesto por el MINSA en el $2018^{[9]}$; 4) se requiere necesariamente de una PS que evalúe la mutación del gen que le confiere R-INH (en este caso Genotype ${ }^{\circledR}$ ) y por lo tanto también a ETO para decidir clínicamente el mejor tratamiento para los pacientes con TB MDR peruanos.

La R-INH es un serio problema de salud pública a nivel nacional. Los tratamientos para la TB MDR deberían ser guiados por los resultados de una PS que evalúen la mutación del gen KatG e InhA para poder brindarle a nuestros pacientes el mejor esquema individualizado de tratamiento.

Contribuciones de autoría: SPS y ACNS concibieron y diseñaron la investigación, recolectaron los datos y redactaron y aprobaron la versión final del artículo. SPS analizó los datos y redacto la tabla; SPS y ACNS revisaron críticamente el artículo. Ambos se responsabilizan por el contenido del artículo y se comprometen a responder adecuadamente las preguntas que pudieran ser necesarias para garantizar la precisión de los datos e integridad de cualquier parte de su investigación.

Financiamiento: autofinanciado.

Potenciales conflictos de interés: los autores declaran no tener conflictos de interés.

\section{ORCID}

Samuel Pecho-Silva, https://orcid.org/0000-0002-7477-9841

Ana C. Navarro-Solsol, https://orcid.org/0000-0001-8983-4324

Tabla 1. Mutación del gen KatG e InhA en 226 aislamientos de pacientes con tuberculosis de EsSalud. Setiembre 2017 - marzo 2018.

\begin{tabular}{|c|c|c|c|c|c|c|}
\hline \multirow{2}{*}{ Valor } & \multirow{2}{*}{ Total } & \multicolumn{2}{|c|}{ Prueba usada } & \multicolumn{3}{|c|}{ Tipo de mutación } \\
\hline & & MODS & Genotype & KatG & $\operatorname{Inh} A$ & KatG / InhA \\
\hline S: INH S: RIF & 38 & 8 & 30 & - & - & - \\
\hline S: INH R: RIF & 20 & 4 & 16 & - & - & - \\
\hline R: INH S: RIF & 66 & 3 & 63 & 33 & 28 & 2 \\
\hline R: INH R: RIF & 126 & 9 & 117 & 83 & 22 & 0 \\
\hline Total & 250 & 24 & 226 & 116 & 50 & 2 \\
\hline
\end{tabular}

S: sensible; R: resistente; INH: isoniazida; RIF: Rifampicina 


\section{REFERENCIAS BIBLIOGRÁFICAS}

1. Seifert M, Catanzaro D, Catanzaro A, Rodwell TC. Genetic mutations associated with isoniazid resistance in Mycobacterium tuberculosis: a systematic review. PLoS One. 2015;10(3):e0119628. doi: 10.1371/ journal.pone.0119628

2. Bollela VR, Namburete EI, Feliciano CS, Macheque D, Harrison $\mathrm{LH}$, Caminero JA. Detection of katG and inhA mutations to guide isoniazid and ethionamide use for drug-resistant tuberculosis. Int J Tuberc Lung Dis. 2016;20(8):1099-104. doi:10.5588/ijtld.15.0864

3. Pecho-Silva S., Navarro-Solsol A, Chiappe-Gonzalez A. Resistencia a isoniacida, un problema de salud pública: historia y tratamiento. Rev Peru Investig Salud. 2019;3(2):81-8. doi: 10.35839/repis.3.2.264

4. Vadwai V, Ajbani K, Jose M, Vineeth VP, Nikam C, Deshmukh M, et al. Can inhA mutation predict ethionamide resistance? Int J Tuberc Lung Dis. 2013;17(1):129-30. doi: 10.5588/ijtld.12.0511

5. Niehaus AJ, Mlisana K, Gandhi NR, Mathema B, Brust JCM. High Prevalence of inhA Promoter Mutations among Patients with Drug-
Resistant Tuberculosis in KwaZulu-Natal, South Africa. PLoS One. 2015;10(9):e0135003. doi: 10.1371/journal.pone.0135003

6. Quispe N, Asencios L, Obregon C, Velásquez GE, Mitnick CD, Lindeborg $\mathrm{M}$, et al. The fourth national anti-tuberculosis drug resistance survey in Peru. Int J Tuberc Lung Dis. 2020;24(2):207-13. doi: 10.5588/ijtld.19.0186

7. Sulis G, Pai M. Isoniazid-resistant tuberculosis: A problem we can no longer ignore. PLoS Med. 2020;17(1):e1003023. doi:10.1371/ journal.pmed.1003023

8. Dean AS, Zignol M, Cabibbe AM, Falzon D, Glaziou P, Cirillo DM, et al. Prevalence and genetic profiles of isoniazid resistance in tuberculosis patients: A multicountry analysis of cross-sectional data. PLoS Med. 2020;17(1):e1003008. doi:10.1371/journal. pmed. 1003008

9. Ministerio de Salud del Perú. Resolución Ministerial 752-2018/ MINSA. Lima, Perú: MINSA; 2018 [citado 20 de junio 2020]. Disponible en: https://cdn.www.gob.pe/uploads/document/ file/186939/Resolucion_Ministerial_752-2018-MINSA.PDF 